IGCS20_1451

\section{PROGNOSTIC FACTORS RELATED TO RECURRENCE AND SURVIVAL OF EARLY STAGE VULVAR SQUAMOUS CELL CARCINOMA}

${ }^{1} \mathrm{Y}$ Wang ${ }^{*},{ }^{2} \mathrm{~K}$ Lindemann, ${ }^{3} \mathrm{~K}$ Bruheim, ${ }^{1} \mathrm{~A}$ Barrameda, ${ }^{4} \mathrm{~B}$ Davidson, ${ }^{1} \mathrm{~T}$ Skeie-Jensen. ${ }^{1}$ Department of Gynaecologic Oncology, Oslo University Hospital-Radium Hospital, Oslo, Norway; ${ }^{2}$ Department of Gynaecologic Oncology, Oslo University Hospital-Radium Hospital, Oslo, Norway and Faculty of Medicine, Institute of Clinical Medicine, University of Oslo, Oslo, Norway; ${ }^{3}$ Department of Oncology, Oslo University Hospital-Radium Hospital, Oslo, Norway; ${ }^{4}$ Department of Pathology, Oslo University Hospital-Radium Hospital, Oslo, Norway and Faculty of Medicine, Institute of Clinical Medicine, University of Oslo, Oslo, Norway

10.1136/ijgc-2020-IGCS.359

Objection To evaluate prognostic factors for recurrence and survival among patients with early stage vulvar squamous cell carcinoma (VSCC).

Methods This is a retrospective study of patients with clinical stage I VSCC who were treated at Oslo University Hospital - Radium hospital between 01.01.2006 and 31.12.2016. Clinicopathological characteristics, treatment and follow-up were extracted from the medical records. Univariate and multivariate analysis were used to identify prognostic factors for recurrence, time to recurrence (TTR) and overall survival (OS). A p-value of $<0.05$ was considered to be statistically significant.

Results 133 patients who underwent primary vulva surgery and evaluation of groin lymph node status were included. The median age was 64 years, and groin lymph node metastases were identified in $22.6 \%$ of patients. The median follow-up time was 67 months (range 5-165). The 5-year recurrence and survival rates were $23.3 \%$ and $72.2 \%$, respectively. In multivariate analysis, the presence of lichen sclerosus and groin lymph node metastasis were independent prognostic factors for recurrence and TTR, with an odds ratio $(95 \% \mathrm{CI})$ of $5.37(2.13-13.53)$ and $2.8(1.17-6.72)$ for recurrence, and a HR (95\% CI) of $2.6(1.35-5.19)$ and 2.2 (1.13-4.26) for TTR, respectively. Age $>70$ years and a history of recurrence were independent prognostic factors for OS, with a HR $(95 \% \mathrm{CI})$ of $3.0(1.58-5.65)$ and 2.1 (1.57$6.15)$, respectively.

Conclusions Patients with lichen sclerosus and groin lymph node metastasis have a higher risk for recurrence and shorter TTR. Patients with age $>70$ years and a history of recurrence have significantly poorer OS.

\section{IGCS20 1452}

\section{PROGNOSTIC FACTORS FOR RECURRENCE IN CARCINOMA ENDOMETRIUM}

'A Prakasan*, 'M Dhas, 'A Kumar, 'S Mathews, 'J Joseph, ${ }^{2} \mathrm{~J}$ Krishna, ${ }^{2} \mathrm{AJV},{ }^{3} \mathrm{SS}$, ${ }^{1} \mathrm{FV}$ James. 'Department of Radiation Oncology, Regional Cancer Centre, India; ${ }^{2}$ Department of Bio statistics and Epidemiology, Regional Cancer Centre, India; ${ }^{3}$ Department of Surgical Oncology, Regional Cancer Centre, India; Prognostic factors for recurrence in Carcinoma Endometrium

\subsection{6/ijgc-2020-IGCS.360}

Objective Incidence of endometrial cancer is on the rise in India. Although there have been significant advances in understanding of endometrial cancer biology, these factors are not yet included in routine management of patients. The aim of the study was to identify prognostic factors for risk stratification and offering judicious adjuvant therapy.

Methods Retrospective analysis of data of patients with carcinoma endometrium registered at Regional Cancer Centre, Thiruvananthapuram from January 2009 to December 2013 was done. Various patient, tumor and treatment related factors were analyzed for its effect on recurrence. Survival estimates were generated using Kaplan -Meier method. Univariate analysis was done using Chi-square and Fisher's exact tests and multivariate analysis was done using Cox regression model. The statistical analysis was done using SSPS software version 11.

Results The median follow up of 642 patients was 95 months (range 3-178 months).There were 432 stage 1 (67\%), 100 stage II ( $15.57 \%), 108$ stage III (16.8\%)and 2 stage IVa patients $(0.3 \%)$. The five-year disease free survival (DFS) was $82.1 \%$. Prognostic factors for DFS on multivariate analysis were age $>60$ years, high grade tumor, advanced stage, deep myometrial invasion, cervical stromal invasion, and negative Progesterone Receptor (PR) status. Cervical stromal invasion, negative PR status, no adjuvant treatment were associated with pelvic failure.

Conclusion The predictive and prognostic factors for Carcinoma endometrium is similar to those published in developed countries.

\section{IGCS20_1453}

\section{CERVICAL CARCINOSARCOMA WITH REMISSION OF MALIGNANT EPITHELIAL COMPONENT BUT PERSISTING STROMAL COMPONENT AFTER RADIOTHERAPY - ONE CASE REPORT}

${ }^{1} \mathrm{Y}$ Sun*, ${ }^{1} \mathrm{~K}$ Hung, ${ }^{1} \mathrm{C}$ Kang, ${ }^{2} \mathrm{~S}$ Chang. . Department of Obstetrics and Gynecology, Chimei Medical Center, Taiwan; ${ }^{2}$ Department of Pathology and Laboratory Medicine, Chimei Medical Center, Taiwan

\subsection{6/ijgc-2020-IGCS.361}

Introduction Carcinosarcomas of the uterine cervix are rare. Herein, we report a woman diagnosed cervical carcinosarcoma with remission of malignant epithelial component but persisting stromal component after radiotherapy.

Case report A 30 y/o woman presented to our hospital with bulky cervical tumor FIGO stage IIIB. The cervical biopsy came from other hospital reported as adenocarcinoma. She was arranged for concurrent chemo radiation. However, Cisplatin was unable to give due to persisting pancyopenia after initiating radiotherapy. Bone marrow biopsy for her persisting pancytopenia disclosed acellular marrow. After completed external beam radiation therapy followed with brachytherapy, there is one cervical mass noted confined within the cervical canal, with biopsy disclosed sarcoma. She underwent abdominal total hysterectomy and bilateral salpingo-oophorectomy. Pathology revealed tumor cells with neuroectodermal differentiation, involving cervix, uterus and ovaries. After taken previous biopsy section together, a carcinosarcoma with heterogenous neuroectodermal component was inferred, while the components of adenocarcinoma have been eradicated by previous radiotherapy and the components with neuroectodermal differentiation survived due to resistance. Unfortunately, chemotherpay was not eligible before bone marrow 